\title{
ISOMORPHISMS OF INFINITE-DIMENSIONAL ANALOGUES OF THE CLASSICAL GROUPS
}

\author{
C. E. RICKART
}

1. Introduction. The classical groups referred to here are the full linear, symplectic, orthogonal, and unitary groups. These are groups of linear transformations operating on a finite-dimensional linear vector space over a (commutative) field. In order to save time, we shall not repeat the usual definitions of the groups but shall proceed directly to definitions ${ }^{1}$ which are meaningful for the infinite- as well as the finite-dimensional case. At the same time, the condition that the coefficient domain be commutative will be relaxed and semi-linear as well as linear transformations will be admitted.

The main objectives in this address are to describe the structure of isomorphisms between two such generalized classical groups and to outline some of the methods used in studying these isomorphisms. It turns out that the isomorphisms are, roughly speaking, induced by isomorphisms between the underlying vector spaces on which the transformations act.

In the case of the full linear group in finite dimensions, this problem (for automorphisms) has been considered by Schreier and van der Waerden [12] ${ }^{2}$ when the coefficient domain is commutative, and by Dieudonné [2] when the coefficient domain is not commutative. Dieudonné has also considered the other classical groups in the finite-dimensional case. Mackey [9] has considered the problem for the multiplicative group of all bounded linear transformations with bounded inverses on an infinite-dimensional real normed linear space. The case studied by Mackey is included in our generalization of the full linear group.

The method of attack employed here is the standard one of investigating the way in which involutions are transformed by the isomorphisms plus an application of the fundamental theorem of projective geometry. However the special methods used in the investigation are refinements of methods introduced by Mackey in the paper mentioned above. The main results outlined are discussed in detail for linear transformations in [10] for the full linear case

An invited addressed delivered before the New York meeting of the Society, October 28, 1950, by invitation of the Committee to Select Hour Speakers for Eastern Sectional Meetings; received by the editors August 6, 1951.

1 These definitions are given in the next section.

2 Numbers in brackets refer to the bibliography at the end of the paper. 
and in $[11]$ for the other cases. ${ }^{3}$ The extension to semi-linear transformations is sketched here for the first time.

2. Definitions. We consider first the notion of dual linear vector spaces as introduced by Dieudonné [4] and Jacobson [8]. Let $\mathfrak{X}$ and $\mathfrak{X}^{*}$ be left and right linear vector spaces respectively over a division ring (skew field or sfield) $\mathcal{D}$. Assume given a bilinear functional $\left(x, x^{*}\right)$ defined on $\mathfrak{X} \times \mathfrak{X}^{*}$ to $\mathcal{D}$ which is nondegenerate in the sense that $\left(x, x^{*}\right)=0$ for all $x$ (resp. all $x^{*}$ ) implies $x^{*}=0$ (resp. $x=0$ ); then $\mathfrak{X}$ and $\mathfrak{X}^{*}$ are said to be $d u a l$ relative to $\left(x, x^{*}\right)$. If $\overline{\mathfrak{X}}$ denotes the right vector space of all linear functionals on $\mathfrak{X}$ and if $(x, \bar{x})$ denotes the value of the functional $\bar{x}$ at $x$, then $\mathfrak{X}$ and $\overline{\mathfrak{X}}$ are evidently dual relative to $(x, \bar{x})$. In general, if $\mathfrak{X}$ and $\mathfrak{X}^{*}$ are dual relative to $\left(x, x^{*}\right)$, then $\left(x, x^{*}\right)$, for fixed $x^{*}$, defines a linear functional on $\mathfrak{X}$. Hence $\mathfrak{X}^{*}$ can be regarded as a subspace of $\overline{\mathfrak{X}}$. Similarly, $\mathfrak{X}$ can be regarded as a subspace of $\overline{\mathfrak{X}}^{*}$. In case $\mathfrak{X}$ is finite-dimensional, we necessarily have $\mathfrak{X}^{*}=\overline{\mathfrak{X}}$.

Let $\mathfrak{X}$ be a left linear vector space over $\mathcal{D}$ and let $T$ be a transformation defined on $\mathfrak{X}$ to $\mathfrak{X}$. The transformation $T$ is said to be semilinear provided it is additive and there exists an automorphism $\alpha \rightarrow \alpha^{\tau}$ of $\mathcal{D}$ such that $(\alpha x) T=\alpha^{\tau}(x T)$ for all $\alpha \in \mathcal{D}$ and $x \in \mathfrak{X}$. Now, if $\mathfrak{X}^{*}$ is dual to $\mathfrak{X}$ relative to $\left(x, x^{*}\right)$, then $\left(x T, x^{*}\right)^{\tau^{-1}}$ defines, for fixed $x^{*}$, a linear functional on $\mathfrak{X}$. If for every $x^{*}$ there exists $y^{*}$ in $\mathfrak{X}^{*}$ such that $\left(x T, x^{*}\right)^{\tau^{-1}}=\left(x, y^{*}\right)$, then $x^{*} T^{*}=y^{*}$ defines a semi-linear transformation $T^{*}$ on $\mathfrak{X}^{*}$ with associated automorphism $\tau^{-1}$ of $\mathcal{D}$. When $T^{*}$ exists, it is uniquely determined and is called the adjoint of $T$. Similarly, $T$ is called the adjoint of $T^{*}$ and we write $T=\left(T^{*}\right)^{*}$. If $T$ and $S$ are semi-linear on $\mathfrak{X}$ with associated automorphisms $\tau$ and $\sigma$ of $\mathcal{D}$, then $T S$ is a semi-linear transformation on $\mathfrak{X}$ with automorphism $\tau \sigma$. Also, if $T^{*}$ and $S^{*}$ exist, then $(T S)^{*}$ exists and is equal to $S^{*} T^{*}$. If $T^{*}$ exists and $T$ has an inverse $T^{-1}$, then $\left(T^{-1}\right)^{*}$ exists and is equal to $\left(T^{*}\right)^{-1}$. We can therefore consider the multiplicative group $\mathcal{L}\left(\mathfrak{X}, \mathcal{D}, \mathfrak{X}^{*}\right)$ of all semi-linear transformations on $\mathfrak{X}$ which have adjoints as well as multiplicative inverses. ${ }^{4}$ These groups and certain subgroups of them provide us with our desired generalizations of the full linear group. Included here will be the group of all linear transformations in $\mathcal{L}\left(\mathfrak{X}, \mathcal{D}, \mathfrak{X}^{*}\right)$. The latter group obviously reduces to the

${ }^{3}$ In the papers $[10,11]$, the group operation is taken as the circle operation, $A \circ B=A+B-A B$, instead of multiplication as is done here. However, the two groups obtained are isomorphic under the mapping $A \rightarrow I-A$, where $I$ is the identity transformation.

${ }^{4}$ Strictly speaking, the notation $\mathcal{L}\left(\mathfrak{X}, \mathcal{D}, \mathfrak{X}^{*}\right)$ should exhibit the bilinear functional $\left(x, x^{*}\right)$. 
classical full linear group if $\mathfrak{X}$ is finite-dimensional and $\mathscr{D}$ is a field. For want of a better terminology we shall refer to the groups considered here as "full linear groups," although they may contain semilinear transformations and need not exhaust $\mathcal{L}\left(\mathfrak{X}, \mathcal{D}, \mathfrak{X}^{*}\right)$.

Next we consider the notion of a self-dual linear vector space. Again let $\mathfrak{X}$ be a left linear vector space over the division ring $\mathcal{D}$. Furthermore, assume given an involution $\alpha \rightarrow \alpha^{*}$ (that is, an antiautomorphism of period two) in $\mathcal{D}$ and a functional $(x, y)$ defined on $\mathfrak{X} \times \mathfrak{X}$ to $\mathscr{D}$ and called a scalar product with the following properties: (1) $(x, y)$ is linear in $x$ for each $y$. (2) $(x, y)=0$ for all $y$ implies $x=0$. (3) $(x, y)=\epsilon(y, x)^{*}$, where $\epsilon= \pm 1$ is a constant independent of $x$ and $y$. Under these conditions $\mathfrak{X}$ is said to be self-dual. In fact, if $\mathfrak{X}^{*}$ denotes a right linear vector space over $\mathcal{D}$ which is identical with $\mathfrak{X}$ except with right multiplication by scalars defined by $x \alpha=\alpha^{*} x$, then $\mathfrak{X}$ and $\mathfrak{X}^{*}$ are dual relative to $(x, y)$. Two vectors $x, y$ in $\mathfrak{X}$ are said to be orthogonal, written $x \perp y$, provided $(x, y)=0$. If $\mathfrak{M}$ is a subspace of $\mathfrak{X}$, then $\mathfrak{M}^{\perp}$ will denote the set of all vectors in $\mathfrak{X}$ each of which is orthogonal to every vector in $\mathfrak{M}$. Evidently $\mathfrak{M}^{\perp}$ is a linear subspace of $\mathfrak{X}$ and is called the orthogonal complement of $\mathfrak{M}$. A subspace $\mathfrak{M}$ is said to be isotropic provided $\mathfrak{M} \cap \mathfrak{M}^{\perp} \neq(0)$. If $\mathfrak{M} \subseteq \mathfrak{M}^{\perp}$, then $\mathfrak{M}$ is said to be totally isotropic. The maximum possible dimension for a totally isotropic subspace of $\mathfrak{X}$ is called the index of $\mathfrak{X}$. A vector $x$ such that $(x, x)=0$ is called an isotropic vector. In general, even when a subspace $\mathfrak{M}$ is nonisotropic (that is, $\mathfrak{M} \cap \mathfrak{M}^{\perp}=(0)$ ), we do not have $\mathfrak{X}=\mathfrak{M} \oplus \mathfrak{M}^{\perp}$. On the other hand, if $\mathfrak{M}$ is finite-dimensional and nonisotropic, it is true that $\mathfrak{X}=\mathfrak{M} \oplus \mathfrak{M}^{\perp}$ [11, Lemma 1.1].

Self-dual spaces fall naturally into two classes. In the first, every vector is isotropic, which implies that $\mathcal{D}$ must be a field with identity mapping as involution and $\epsilon=-1$. These spaces are called symplectic. On the other hand, if there exist nonisotropic vectors in the space, a trivial modification of the involution and scalar product yields $\epsilon=1$. In this case the space is called unitary. It will be assumed hereafter that the self-dual spaces considered are either symplectic or unitary (that is, if $\mathfrak{X}$ is not symplectic, then $\epsilon=1$ ).

Now let $T$ be a semi-linear transformation on the self-dual space $\mathfrak{X}$ with $\tau$ as the associated automorphism of $\mathcal{D}$. It is natural to call a second transformation $T^{*}$ on $\mathfrak{X}$ the adjoint of $T$ provided $(x T, y)$ $=\left(x, y T^{*}\right)^{r}$ for all $x$ and $y$ [7]. If $T^{*}$ exists, then it is unique and is a semi-linear transformation with $\alpha \rightarrow \alpha^{* \tau^{-1}} *$ as the associated automorphism of $\mathcal{D}$. Suppose in addition that $T$ has the property $T^{*}=T^{-1}$. Since $T^{-1}$ is a semi-linear transformation with automorphism $\tau^{-1}$, it follows that $\tau^{-1}={ }^{*} \tau^{-1 *}$, or what amounts to the same thing, $\tau$ com- 
mutes with the involution $\left(\alpha^{* *}=\alpha^{* r}\right.$, for all $\left.\alpha\right)$. Furthermore, $(x T, y T)=(x, y)^{\tau}$ for all $x$ and $y$. The set of all semi-linear transformations on $\mathfrak{X}$ such that $T^{*}=T^{-1}$ constitutes a group under multiplication which will be denoted by $\mathcal{L}^{*}(\mathfrak{X}, \mathscr{D})$, in the general self-dual case, and by $\mathcal{L}_{S}^{*}(\mathfrak{X}, \mathscr{D})$ or $\mathcal{L}_{U}^{*}(\mathfrak{X}, \mathscr{D})$ according as $\mathfrak{X}$ is symplectic or unitary. These groups and certain subgroups of them constitute the desired generalizations of the classical symplectic and unitary groups. In the unitary case when $\mathcal{D}$ is a field with involution $\alpha^{*} \equiv \alpha$, we obtain generalizations of the classical orthogonal groups which may be denoted by $\mathcal{L}_{0}^{*}(\mathfrak{X}, \mathcal{D})$. Thus, for our purposes, the orthogonal groups are included among the unitary groups so do not require separate treatment.

In order to simplify our discussion, we shall assume throughout that the vector spaces involved are infinite-dimensional, although everything goes through without change for sufficiently large finite dimensions. It will also be necessary for us to restrict attention to characteristic different from two and, in the unitary case when the index is not zero, to avoid the finite fields $G F(3)$ and $G F(9)$ as coefficients. If the groups considered contain only linear transformations, then the exclusion of the field $G F(9)$ in the unitary case can be dropped. These are the only restrictions imposed in the discussion which follows.

3. Involutions. Let $\mathfrak{X}$ be a left linear vector space over the division ring $\mathcal{D}$ and let $T$ be a semi-linear transformation on $\mathfrak{X}$ for which $T^{2}=I$. Such transformations are called involutions and play an important role in the proofs of the isomorphism theorems to be stated below. Observe that the automorphism $\tau$ of $\mathcal{D}$ associated with an involution $T$ is of period two $\left(\tau^{2}=1\right)$. Since $\mathfrak{X}$ is not of characteristic two, every involution $T$ determines a unique direct sum decomposition $\mathfrak{X}=\mathfrak{M} \oplus \mathfrak{N}$ of the additive group of $\mathfrak{X}$ such that $x T=x$ for $x$ in $\mathfrak{M}$ and $x T=-x$ for $x$ in $\mathfrak{N}$; that is, $T$ coincides with the identity transformation $I$ on $\mathfrak{M}$ and with $-I$ on $\mathfrak{N}$. The components $\mathfrak{M}$ and $\mathfrak{N}$ are called the subspaces of $T$ although they are linear subspaces in $\mathfrak{X}$ if, and only if, $T$ is a linear transformation. Observe that $\mathfrak{M}=\mathfrak{X}(I+T)$ and $\mathfrak{N}=\mathfrak{X}(I-T)$ and, for $x$ in $\mathfrak{X}$, the decomposition $x=m+n$ with $m$ in $\mathfrak{M}$ and $n$ in $\mathfrak{N}$ is given by $m=(x+x T) / 2$ and $n=(x-x T) / 2$. It is easy to verify that an arbitrary additive transformation $Z$ commutes with the involution $T$ if, and only if, $\mathfrak{M} Z \subseteq \mathfrak{M}$ and $\mathfrak{N} Z \subseteq \mathfrak{N}$.

Let $T$ be a linear involution with subspaces $\mathfrak{M}$ and $\mathfrak{R}$. Then $T$ is called minimal if $\mathfrak{R}$ is one-dimensional, maximal if $\mathfrak{M}$ is one-dimensional, and extremal if either $\mathfrak{M}$ or $\mathfrak{N}$ is one-dimensional. Note that $T$ is maximal if, and only if, $-T$ is minimal. A linear involution belongs 
to $\mathcal{L}\left(\mathfrak{X}, \mathcal{D}, \mathfrak{X}^{*}\right)$ and is minimal if, and only if, it has the form $x T$ $=x-2\left(x, v^{*}\right) u$, where $u \in \mathfrak{X}, v^{*} \in \mathfrak{X}^{*}$, and $\left(u, v^{*}\right)=1$. The one-dimensional subspace ${ }^{5}$ of $T$ is $[u]$ and the other subspace of $T$ is a maximal linear subspace of $\mathfrak{X}$ equal to the zero manifold of the functional $v^{*}$ and is thus determined uniquely by the one-dimensional subspace $\left[v^{*}\right]$ of $\mathfrak{X}^{*}$. In this way there is associated with each extremal involution in $\mathcal{L}\left(\mathfrak{X}, \mathscr{D}, \mathfrak{X}^{*}\right)$ a pair of one-dimensional subspaces $[u]$ in $\mathfrak{X}$ and $\left[v^{*}\right]$ in $\mathfrak{X}^{*}$ such that $\left(u, v^{*}\right)=1$. Conversely, to each such pair $[u],\left[v^{*}\right]$ there corresponds two extremal involutions (a minimal involution and its negative) in $\mathcal{L}\left(\mathfrak{X}, \mathcal{D}, \mathfrak{X}^{*}\right)$ having $[u]$ and the zero manifold of $v^{*}$ as subspaces. Evidently there are many extremal involutions having a common subspace; however two such involutions commute if, and only if, they have the same subspaces and are thus equal except for sign.

If $\mathfrak{X}$ is a self-dual space and $T$ is a linear involution such that $T^{*}=T$, then $T$ is in $\mathcal{L}^{*}(\mathfrak{X}, \mathcal{D})$ and the corresponding decomposition of $\mathfrak{X}$ has the form $\mathfrak{X}=\mathfrak{M} \oplus \mathfrak{M}^{\perp}$. Conversely, if $\mathfrak{X}=\mathfrak{M} \oplus \mathfrak{M}^{\perp}$ and $T$ is an involution with subspaces $\mathfrak{M}$ and $\mathfrak{M}^{\perp}$, then $T$ is in $\mathcal{L}^{*}(\mathfrak{X}, \mathcal{D})$. It is obvious that the subspaces associated with a linear involution in $\mathcal{L}^{*}(\mathfrak{X}, \mathcal{D})$ must be nonisotropic. An involution in $\mathcal{L}^{*}(\mathfrak{X}, \mathcal{D})$ is minimal if, and only if, it has the form $x T=x-2(x, u)(u, u)^{-1} u$, where $u$ is a fixed element of $\mathfrak{X}$. Hence $\mathcal{L}_{s}^{*}(\mathfrak{X}, \mathcal{D})$ contains no extremal involutions according to our definition. On the other hand, although a symplectic space contains no one-dimensional nonisotropic subspaces, it is true that there exist many two-dimensional subspaces which are nonisotropic. In fact, every isotropic vector in an arbitrary self-dual space can be embedded in a nonisotropic two-dimensional subspace. Since $\mathfrak{X}=\mathfrak{M} \oplus \mathfrak{M}^{\perp}$, for $\mathfrak{M}$ a finite-dimensional nonisotropic subspace of $\mathfrak{X}$, it follows that $\mathcal{L}_{S}^{*}(\mathfrak{X}, \mathcal{D})$ contains linear involutions with two-dimensional subspaces. Therefore, if $T$ is a linear involution in $\mathcal{L}_{S}^{*}(\mathfrak{X}, \mathcal{D})$ with subspaces $\mathfrak{M}$ and $\mathfrak{M}^{\perp}(x T=x$ for $x \in \mathfrak{M}$ and $x T=-x$ for $x \in \mathfrak{M}^{\perp}$ ), then it is appropriate to define $T$ to be maximal or minimal according as $\mathfrak{M}$ or $\mathfrak{M}^{\perp}$ is two-dimensional and extremal in either case.

In order to handle nonlinear involutions a reduction, which is suggested by an argument used by Dieudonné $[2$, p. 9] in a slightly different situation, is required. Let $\mathfrak{X}$ be a left linear vector space over $\mathcal{D}$ and let $T$ be a nonlinear involution in $\mathfrak{X}$ with associated automorphism $\tau$ of $\mathcal{D}$; then $\tau \neq 1$ and $\tau^{2}=1$. Define $\mathcal{D}_{\tau}$ as the set of all $\xi$ in $\mathscr{D}$ such that $\xi^{\tau}=\xi$ and $\mathcal{D}_{\tau}^{\prime}$ as the set of all $\eta$ in $\mathcal{D}$ such that $\eta^{\tau}=-\eta$. It is

${ }^{5}$ The one-dimensional subspace of $\mathfrak{X}$ which contains a nonzero vector $u$ will be denoted by $[u]$. 
obvious that $\mathscr{D}_{\tau}$ is a division subring of $\mathcal{D}$ and that $\mathscr{D}_{\tau} \cap \mathcal{D}_{\tau}^{\prime}=(0)$. Also $\mathcal{D}=\mathcal{D}_{\tau} \oplus \mathcal{D}_{\tau}^{\prime}$; in fact, for every $\alpha$ in $\mathcal{D}, \alpha=\xi+\eta$, where $\xi$ $=\left(\alpha+\alpha^{\tau}\right) / 2, \eta=\left(\alpha-\alpha^{\tau}\right) / 2, \xi$ is in $\mathscr{D}_{\tau}$, and $\eta$ is in $\mathscr{D}_{\tau}^{\prime}$. If $\eta$ is an arbitrary nonzero element of $\mathscr{D}_{r}^{\prime}$, then it is easy to see that $\mathscr{D}_{\tau}^{\prime}=\eta \mathcal{D}_{\tau}$ $=\mathcal{D}_{\tau} \eta$ which shows that $\mathcal{D}$ has (right and left) dimension two over $\mathcal{D}_{\tau}$. Now $\mathfrak{X}$ can be regarded as a linear vector space over $\mathcal{D}_{\tau}$ in which $T$ becomes a linear involution. Hence the subspaces $\mathfrak{M}$ and $\mathfrak{N}$ of $T$ are linear subspaces of $\mathfrak{X}$ over $\mathcal{D}_{\tau}$. Let $\eta$ be a fixed nonzero element of $\mathcal{D}_{\tau}^{\prime}$. Then the mapping $x \rightarrow \eta x$ is a one-to-one mapping of $\mathfrak{M}$ onto $\mathfrak{R}$ which is semi-linear with respect to $\mathscr{D}_{\tau}$. Thus $\mathfrak{M}$ and $\mathfrak{N}$ have the same dimension over $\mathcal{D}_{\tau}$, which is equal to the dimension of $\mathfrak{X}$ over $\mathcal{D}$. If $\mathfrak{X}$ is self-dual and $T$ is in $\mathcal{L}^{*}(\mathfrak{X}, \mathcal{D})$, then $\alpha^{* \tau}=\alpha^{* *}$, for all $\alpha$ in $\mathcal{D}$, so that $\mathscr{D}_{\tau}^{*}=\mathscr{D}_{\tau}$. In other words, $\xi \rightarrow \xi^{*}$ is an involution in $\mathcal{D}_{\tau}$. Moreover $\mathfrak{X}$, as a linear vector space over $\mathcal{D}_{\tau}$, is self-dual relative to the new scalar product $[x, y]=\left[(x, y)+(x, y)^{r}\right] / 2$. Clearly $T^{*}(=T)$ is the adjoint of $T$ with respect to the scalar product $[x, y]$, which implies that $\mathfrak{N}$ is the orthogonal complement of $\mathfrak{M}$ in $\mathfrak{X}$ with respect to $[x, y]$. Since $(x T, y T)=(x, y)^{r}$ for all $x, y$ in $\mathfrak{X}$, it follows that $(x, y)^{r}$ $=(x, y)$ for $x, y$ both in either $\mathfrak{M}$ or $\mathfrak{N}$. Hence the two scalar products $(x, y)$ and $[x, y]$ coincide in $\mathfrak{M}$ and $\mathfrak{R}$. The above discussion, in a sense, reduces the study of nonlinear involutions to the study of linear involutions and makes available methods based on linearity of the subspaces of the involution.

The next problem here is to give a group theoretical characterization of extremal involutions. First let $\mathcal{T}$ denote an arbitrary set of involutions in the group under consideration and denote by $c(\mathcal{G})$ the set of all those involutions in the group which commute with each element of $\mathcal{T}$. Now let $T$ be an arbitrary involution in the group and denote by $\nu_{T}$ the maximum number of elements which can occur in $c\left(c\left(T, T^{\prime}\right)\right)$ where $T^{\prime}$ is any involution which commutes with $T$. Also denote by $\nu$ the maximum value which $\nu_{T}$ can have. The following lemma, whose proof will be omitted, enables one to evaluate the quantities $\nu$ and $\nu_{T}$.

Lemma 1. Let $T_{i}$ be an involution with subspaces $\mathfrak{M}_{i}, \mathfrak{N}_{i}(i=1,2)$ and assume that $T_{1}$ and $T_{2}$ commute. Set $\mathfrak{P}_{1}=\mathfrak{M}_{1} \cap \mathfrak{M}_{2}, \mathfrak{P}_{2}=\mathfrak{M}_{1} \cap \mathfrak{N}_{2}$, $\mathfrak{P}_{3}=\mathfrak{N}_{1} \cap \mathfrak{M}_{2}, \mathfrak{P}_{4}=\mathfrak{N}_{1} \cap \mathfrak{N}_{2}$. Observe that $\mathfrak{X}=\mathfrak{B}_{1} \oplus \mathfrak{P}_{2} \oplus \mathfrak{P}_{3} \oplus \mathfrak{P}_{4}$ and denote by $P_{i}$ the projection of $\mathfrak{X}$ onto $\mathfrak{P}_{i}$ determined by this decomposition of $\mathfrak{X}$. Then every $T$ in $c\left(c\left(T_{1}, T_{2}\right)\right)$ is of the form $T=\sum \epsilon_{i} P_{i}$ where each $\epsilon_{i}= \pm 1$.

If the $T_{1}$ and $T_{2}$ in Lemma 1 are linear involutions, then each $\mathfrak{P}_{i}$ is a linear subspace of $\mathfrak{X}$ and $\sum \epsilon_{i} P_{i}$ is a linear involution which belongs 
to the group under consideration for every choice of $\epsilon_{i}= \pm 1$. If none of the $P_{i}$ is zero, it follows in this case that the number of distinct elements in $c\left(c\left(T_{1}, T_{2}\right)\right)$ is equal to 16 . It is not difficult to construct linear involutions $T_{1}$ and $T_{2}$ in the group such that no $P_{i}$ is zero; hence we obtain $\nu=16$. If either $T_{1}$ or $T_{2}$ is not linear, then in order for $\sum \epsilon_{i} P_{i}$ to be a semi-linear transformation the coefficients $\epsilon_{i}$ can no longer be independentlychosen; in fact, it turns out that $c\left(c\left(T_{1}, T_{2}\right)\right)$ contains only the elements $\pm I, \pm T_{1}, \pm T_{2}, \pm T_{1} T_{2}$. This same result holds if either $T_{1}$ or $T_{2}$ is extremal or if either $T_{1}$ or $T_{2}$ is equal to $\pm I$. These remarks lead to the following lemma.

Lemma 2. (i) $T$ linear but not extremal is equivalent to $\nu_{T}=\nu$. (ii) $T$ either extremal or nonlinear is equivalent to $\nu_{T}=\nu / 2$. (iii) $T$ equal to $\pm I$ is equivalent to $\nu_{T}=\nu / 4$.

The above lemma gives a group-theoretic characterization of nontrivial linear involutions which are not extremal but does not distinguish between extremal involutions and nonlinear involutions. On the other hand, it is not difficult to see that any extremal involution can be written as a product of two commuting linear non-extremal involutions. This remark plus Lemma 2 gives the desired characterization of extremal involutions.

Lemma 3. An involution $T$ is extremal if, and only if, $\nu_{T}=\nu / 2$ and there exist involutions $T_{1}$ and $T_{2}$, with $\nu_{T_{1}}=\nu_{T_{2}}=\nu$, such that $T=T_{1} T_{2}$ $=T_{2} T_{1}$.

This criterion for extremal involutions is valid for any subgroups of $\mathcal{L}\left(\mathfrak{X}, \mathcal{D}, \mathfrak{X}^{*}\right)$ or $\mathcal{L}^{*}(\mathfrak{X}, \mathcal{D})$ provided only that the subgroups contain all minimal involutions. Such subgroups need not contain $-I$ in which case it turns out that $\nu=8$ instead of 16 . Included here are the groups of linear transformations for which the above discussion becomes considerably simpler. The criterion also applies for finite dimensions at least equal to ten in the symplectic case and to five in the other cases. However, it breaks down for lower dimensions since an extremal involution can no longer be written as a product of nonextremal linear involutions. On the other hand, if the groups contain only linear transformations, then Lemma 2 gives a criterion for extremal involutions which applies for dimensions greater than six in the symplectic case and greater than three in the other cases. For lower dimensions, the linear involutions are all extremal.

We pass now to the isomorphism theorems and consider the full linear case first.

4. The full linear case. Consider two groups of type $\mathcal{L}\left(\mathfrak{X}, \mathcal{D}, \mathfrak{X}^{*}\right)$ 
and $\mathcal{L}\left(\mathfrak{Y}, \mathcal{E}, \mathfrak{Y}^{*}\right)$ and let $G$, $\mathfrak{F}$ be subgroups of $\mathcal{L}\left(\mathfrak{X}, \mathcal{D}, \mathfrak{X}^{*}\right), \mathcal{L}\left(\mathfrak{Y}, \mathcal{E}, \mathfrak{Y}^{*}\right)$ respectively which contain all minimal involutions in each case. Also assume given an isomorphic mapping $G \rightarrow g(G)$ of $G$ onto $\mathfrak{H C}$ and denote by $\varepsilon_{0}$ the multiplicative group consisting of the nonzero elements of $\varepsilon$. The following theorem gives the form of $\mathfrak{g}$ in this situation. In order to state the theorem, some definitions are required. First, a one-to-one mapping $\Phi$ of $\mathfrak{X}$ onto $\mathfrak{V}$ is called an isomorphism of $\mathfrak{X}$ onto $\mathfrak{Z}$ if it is additive and there exists an isomorphism $\alpha \rightarrow \alpha^{\phi}$ of $\mathcal{D}$ onto $\mathcal{E}$ such that $(\alpha x) \Phi=\alpha^{\phi}(x \Phi)$ for all $\alpha$ in $\mathcal{D}$ and $x$ in $\mathfrak{X}$. Similarly, a one-to-one mapping $\Phi$ of $\mathfrak{X}$ onto $\mathfrak{Y}^{*}$ is called an isomorphism of $\mathfrak{X}$ onto $\mathfrak{Y}^{*}$ if it is additive and there exists an antiisomorphism $\phi$ of $\mathcal{D}$ onto $\mathcal{E}$ such that $(\alpha x) \Phi=(x \Phi) \alpha^{\phi}$. Next, a mapping $G \rightarrow \chi(G)$ of $G$ into $\varepsilon_{0}$ is called a crossed character of $G$ in $\varepsilon_{0}$ [1] if there exists a homomorphism $G \rightarrow \sigma(G)$ of $G$ into the group of automorphisms of $\varepsilon_{0}$ such that $\chi\left(G_{1} G_{2}\right)=\chi\left(G_{1}\right)^{\sigma^{\left(G_{2}\right)}} \chi\left(G_{2}\right)$ for all $G_{1}, G_{2}$ in $G$. If $\sigma(G)=1$ for all $G$, then $\chi(G)$ is called a character of $G$ in $\varepsilon_{0}$.

Theorem I. The isomorphism $\mathfrak{g}$ has, for all $G$ in $G$, one of the two forms: (i) $\mathfrak{g}(G)=\chi(G) \Phi^{-1} G \Phi$, (ii) $\mathfrak{g}(G)=\left(\Phi^{-1} G \Phi \chi(G)\right)^{*}$, where $\Phi$ in case (i) is an isomorphism of $\mathfrak{X}$ onto $\mathfrak{Z}$ and in case (ii) is an isomorphism of $\mathfrak{X}$ onto $\mathfrak{Y}^{*}$ and $\chi(G)$ is a crossed character ${ }^{6}$ of $G$ in $\varepsilon_{0}$.

COROLlary. If the groups consist of linear transformations, then $\chi(G)$ is a character of $G$ in the center of $\varepsilon_{0}$.

The proof of Theorem I is too long to be given in detail here; $;^{7}$ however it is possible to outline the main ideas which rely heavily on methods introduced by Mackey [9].

By the group-theoretic characterization of extremal involutions given in Lemma 3 of the preceding section, it follows that $g$ establishes a one-to-one correspondence between extremal involutions in $G$ and in $\mathcal{H}$. In order to use this fact it is necessary to formulate a group-theoretic criterion for two (noncommutative) extremal involutions to have a common subspace. This is given in the next lemma, whose proof will be omitted.

Lemma 4. Two noncommutative extremal involutions $T_{1}$ and $T_{2}$ have a common subspace if, and only if, $c\left(c\left(T_{1}, T_{2}\right)\right)=c\left(c\left(U_{1}, U_{2}\right)\right)$ for every pair $U_{1}, U_{2}$ of noncommutative extremal involutions in $c\left(c\left(T_{1}, T_{2}\right)\right)$.

Now let $[x]$ be an arbitrary one-dimensional subspace of $\mathfrak{X}$ and

- The homomorphism $G \rightarrow \sigma(G)$ of $G$ into the group of automorphisms of $\varepsilon_{0}$ is given by $\sigma(G)=\gamma^{\prime}$ where $\gamma^{\prime}$ is the automorphism of $\varepsilon$ associated with the semi-linear transformation $\mathfrak{g}(G)$.

${ }^{7}$ See $[10]$ for a detailed proof in the case of groups of linear transformations. 
consider two noncommutative extremal involutions $T_{1}, T_{2}$ which have $[x]$ as a common subspace. Then, by Lemma 4 , the extremal involutions $\mathfrak{g}\left(T_{1}\right), \mathfrak{g}\left(T_{2}\right)$ also have a common subspace which, however, may or may not be one-dimensional. On the other hand, it is possible to show that the subspace common to $\mathfrak{g}\left(T_{1}\right)$ and $\mathfrak{g}\left(T_{2}\right)$ is independent of the choice of $T_{1}, T_{2}$ and is, or is not, one-dimensional independently of $[x]$. In case the subspace common to $g\left(T_{1}\right)$ and $\mathfrak{g}\left(T_{2}\right)$ is one-dimensional, we denote it by $[x]^{\prime}$ and so obtain a one-to-one correspondence $[x] \leftrightarrow[x]^{\prime}$ between the one-dimensional subspaces of $\mathfrak{X}$ and those of $\mathfrak{Y}$. In the other case, the subspace common to $\mathfrak{g}\left(T_{1}\right)$ and $\mathfrak{g}\left(T_{2}\right)$ is a maximal subspace of $\mathfrak{Y}$ which is the zero manifold of a linear functional $y^{*}$ in $\mathfrak{Y}^{*}$. In this case we define $[x]^{\prime}=\left[y^{*}\right]$ and obtain a one-to-one correspondence between the one-dimensional subspaces of $\mathfrak{X}$ and those of $\mathfrak{Y}^{*}$. Now an application of the fundamental theorem of projective geometry gives an isomorphism $\Phi$, of $\mathfrak{X}$ onto $\mathfrak{Y}$ in the first case and of $\mathfrak{X}$ onto $\mathfrak{Y}^{*}$ in the second case, such that $[x \Phi]=[x]^{\prime}$ for all $x$ in $\mathfrak{X}$. The isomorphism $\Phi$ has the additional property that it maps the subspaces of an extremal involution $T$ in $G$ onto the subspaces of $\mathfrak{g}(T)$ or $\mathfrak{g}(T)^{*}$ according as $\Phi$ maps $\mathfrak{X}$ onto $\mathfrak{Y}$ or $\mathfrak{Y}^{*}$. It follows that if $T$ is an extremal involution, then $\mathfrak{g}(T)$ $= \pm \Phi^{-1} T \Phi$ in the first case and $\mathfrak{g}(T)= \pm\left(\Phi^{-1} T \Phi\right)^{*}$ in the second. Thus $\mathfrak{g}(T)$ has the indicated form for extremal involutions. That $\mathfrak{g}(G)$ has the desired form for all $G$ is a consequence of the obvious fact that the minimal involutions constitute an invariant subset of $\mathcal{L}\left(\mathfrak{X}, \mathcal{D}, \mathfrak{X}^{*}\right)$. This can be seen in the following way for the case in which $\Phi$ maps $\mathfrak{X}$ onto $\mathfrak{Y}$. Define $G^{\sigma}=\Phi \mathfrak{g}(G) \Phi^{-1}$. Then $G \rightarrow G^{\sigma}$ is an isomorphism of $G$ onto another subgroup of $\mathcal{L}\left(\mathfrak{X}, \mathscr{D}, \mathfrak{X}^{*}\right)$ such that $T^{\sigma}= \pm T$ when $T$ is a minimal involution. Furthermore, if $T$ is minimal, then so also is $G T G^{-1}$ for arbitrary $G$ in $\mathcal{L}\left(\mathfrak{X}, \mathcal{D}, \mathfrak{X}^{*}\right)$. Hence $G^{\sigma} T\left(G^{\sigma}\right)^{-1}=G T G^{-1}$ for all $G$ in $G$. It follows that $x G T=x G$ if, and only if, $x G^{\sigma} T=x G^{\sigma}$. On the other hand, if $x_{1}$ and $x_{2}$ are linearly independent elements of $\mathfrak{X}$, then there exists a minimal involution $T$ such that $x_{1} T=x_{1}$ and $x_{2} T \neq x_{2}$. Therefore $x G^{\sigma}$ and $x G$ are linearly dependent for all $x$. This implies that $G^{\sigma}$ is equal to a scalar multiple of $G$. Returning to $\mathfrak{g}(G)$, we obtain case (i) of the theorem. That $\chi(G)$ is a crossed character in $\mathcal{E}_{0}$ is immediate from the fact that $G \rightarrow \mathrm{g}(G)$ is a group isomorphism. The case in which $\Phi$ maps $\mathfrak{X}$ onto $\mathfrak{Y}^{*}$ is treated similarly and leads to case (ii) of the theorem.

The above theorem is true and the proof outlined here applies also in the finite-dimensional case provided only that the vector spaces have dimension at least equal to five. If the groups contain only linear transformations, then the minimum dimension five can be 
replaced by three. The finite-dimensional case for groups of linear transformations is due essentially to Dieudonné $[2, \mathrm{pp} .7,15]$ who considered automorphisms rather than isomorphisms. Dieudonné also disposes of the case when the characteristic is equal to two. Hua [6, p. 101] extended the Dieudonné results to dimension two. When the coefficient domain is a (commutative) field, these cases follow from results of Schreier and van der Waerden [12] whose methods are quite different from those used here. The following infinitedimensional case has been considered by Mackey [9]. Let $\mathfrak{X}$ be an infinite-dimensional real normed linear space and take the dual space $\mathfrak{X}^{*}$ as the space of all bounded linear functionals on $\mathfrak{X}$. Then $\mathcal{L}\left(\mathfrak{X}, \mathcal{D}, \mathfrak{X}^{*}\right)$ is the group of all bounded linear transformations on $\mathfrak{X}$ which have inverses. Mackey [9] proved that isomorphism of two groups of this type implies isomorphism of the vector spaces but did not obtain the form of the group isomorphism in terms of the vector space isomorphism. Construction of the isomorphism of the vector space is, of course, the main part of the proof of Theorem I and the methods introduced by Mackey carry over without essential modification to the general case. As a matter of fact, Mackey had already observed [9] that his discussion could be applied to the group of all linear transformations in $\mathcal{L}\left(\mathfrak{X}, \mathcal{D}, \mathfrak{X}^{*}\right)$ when $\mathcal{D}$ is the real numbers. He calls the dual pair $\mathfrak{X}, \mathfrak{X}^{*}$ a linear system.

5. The symplectic and unitary cases. Consider two groups of type $\mathcal{L}^{*}(\mathfrak{X}, \mathcal{D})$ and $\mathcal{L}^{*}(\mathfrak{Y}, \mathcal{E})$ both of which are either symplectic or unitary and let $G$, F be subgroups of $\mathcal{L}^{*}(\mathfrak{X}, \mathcal{D}), \mathcal{L}^{*}(\mathfrak{Y}, \mathcal{E})$ respectively which contain all of the minimal involutions in each case. Also assume given an isomorphic mapping $G \rightarrow \mathfrak{g}(G)$ of $G$ onto $\mathfrak{F}$ and denote by $\varepsilon_{0}^{*}$ the multiplicative group of elements $\mu$ in $\varepsilon_{\text {such that }}$ $\mu \mu^{*}=1$. The following theorem gives the form of $g$ in this situation.

Theorem II. The isomorphism $\mathfrak{g}$ has the form $\mathfrak{g}(G)=\chi(G) \Phi^{-1} G \Phi$, where $\Phi$ is an isomorphism of $\mathfrak{X}$ onto $\mathfrak{Y}$ and $\chi(G)$ is a crossed character ${ }^{6}$ of $G$ in $\varepsilon_{0}^{*}$. If $\phi$ denotes the isomorphism of $\mathcal{D}$ onto $\mathcal{E}$ associated with $\Phi$, then there exists a constant $\rho$ in $\mathcal{E}$ such that $\rho^{*}=\rho, \alpha^{\phi *}=\rho^{-1} \alpha^{* \phi} \rho$ for all $\alpha$ in $\mathcal{D}$, and $\left(x_{1} \Phi, x_{2} \Phi\right)=\left(x_{1}, x_{2}\right)^{\phi} \rho$ for all $x_{1}, x_{2}$ in $\mathfrak{X}$.

CoRollary. In the symplectic and orthogonal cases $\chi(G)= \pm 1$ for each $G$. If $G$ and 30 consist only of linear transformations, then $\chi(G)$ is in the center of $\mathcal{E}$ so that in case $\mathcal{G}$ also coincides with its commutator subgroup,$^{8}$ we have $\chi(G) \equiv 1$.

8 This is so, for example, for the finite-dimensional symplectic group of linear transformations [3, p. 12]. 
We outline ${ }^{9}$ the proof of Theorem II which parallels that of Theorem I. The first step is to construct a one-to-one corrrespondence between the one-dimensional subspaces of $\mathfrak{X}$ and of $\mathfrak{Y}$. Since $\mathfrak{g}$ establishes a one-to-one correspondence between extremal involutions in $G$ and $\mathcal{F}$, one obtains immediately (in the unitary case) a one-to-one correspondence $[x] \leftrightarrow[x]^{\prime}$ between the nonisotropic one-dimensional subspaces of $\mathfrak{X}$ and of $\mathfrak{Y}$. When the index is zero, the first step is thus completed. On the other hand, if $[x]$ is isotropic, choose nonisotropic two-dimensional subspaces $\mathfrak{M}_{1}, \mathfrak{M}_{2}$ such that $\mathfrak{M}_{1} \cap \mathfrak{M}_{2}=[x]$ and let $T_{1}, T_{2}$ be involutions in $G$ with $\mathfrak{M}_{1}, \mathfrak{M}_{2}$ as subspaces. In the symplectic case, $T_{1}$ and $T_{2}$ are extremal so that $\mathrm{g}\left(T_{1}\right)$ and $\mathrm{g}\left(T_{2}\right)$ are also extremal. Moreover the two-dimensional subspaces of $\mathfrak{g}\left(T_{1}\right)$ and $\mathfrak{g}\left(T_{2}\right)$ intersect in a one-dimensional subspace $[x]^{\prime}$. This fact is ensured by the following lemma.

Lemma 5. Let $T_{1}$ and $T_{2}$ be extremal symplectic involutions. Then a necessary and sufficient condition for the two-dimensional subspaces of $T_{1}$ and $T_{2}$ to intersect in a one-dimensional subspace is that $c\left(c\left(T_{1}, T_{2}\right)\right)$ $=c\left(c\left(U_{1}, U_{2}\right)\right)$ for every pair $U_{1}$ and $U_{2}$ of noncommutative extremal involutions in $c\left(c\left(T_{1}, T_{2}\right)\right)$.

It turns out that $[x]^{\prime}$ is independent of the choice of $T_{1}, T_{2}$ so that $[x] \leftrightarrow[x]^{\prime}$ gives the desired one-to-one correspondence in this case. In the unitary case, $T_{1}$ and $T_{2}$ are not extremal but each can be written as a product of two extremal involutions. This implies that $\mathfrak{g}\left(T_{1}\right)$ and $\mathfrak{g}\left(T_{2}\right)$ each has a two-dimensional subspace and an additional argument shows that these two-dimensional subspaces intersect in an isotropic one-dimensional subspace $[x]^{\prime}$ which is independent of the choice of $\mathfrak{M}_{1}$ and $\mathfrak{M}_{2}$. The resulting correspondence between isotropic one-dimensional subspaces plus that already obtained for the nonisotropic one-dimensional subspaces provides us with the desired one-to-one correspondence $[x] \leftrightarrow[x]^{\prime}$ between one-dimensional subspaces of $\mathfrak{X}$ and of $\mathfrak{Y}$. In both the symplectic and unitary cases it turns out that the correspondence $[x] \leftrightarrow[x]^{\prime}$ preserves orthogonality. The fundamental theorem of projective geometry thus gives an orthogonality preserving isomorphism $\Phi$ of $\mathfrak{X}$ onto $\mathfrak{Y}$ such that $[x \Phi]=[x]^{\prime}$. The fact that $\Phi$ preserves orthogonality implies the properties mentioned in the last statement of the theorem. The representation of $\mathfrak{g}(G)$ is obtained by an argument similar to that given in the full linear case.

The above theorem is true and the proof outlined applies to the finite-dimensional case provided that the vector spaces have dimen-

${ }^{\ominus}$ See $\lceil\mathbf{1 1}\rceil$ for a detailed proof for groups of linear transformations. 
sions at least equal to ten. In the unitary case with zero index, it is enough to have the dimensions at least equal to five. If the groups contain only linear transformations, then these minimum dimensions can be replaced by six and three respectively. For the finitedimensional symplectic case and automorphisms of groups of linear transformations, Hua [5] has proved the above theorem for dimensions at least equal to two. Hua's result was extended by Dieudonné $[2$, p. 39] to include the case of characteristic two. Dieudonné [2, pp. 51, 79, 82] has also considered the finite-dimensional orthogonal and unitary cases (characteristic not equal to two) for automorphisms of groups of linear transformations when the dimension is at least equal to three and the index is different from zero. However, in the unitary case, he restricts the coefficient domain to the following two special instances: (1) the generalized quaternions (reflexive sfield $[2$, p. 81]) with involution equal to the conjugate and (2) a field $K$ which is a separable extension of degree two over a field $K_{0}$ with involution in $K$ equal to that automorphism of $K$ over $K_{0}$ different from the identity. In the latter instance, the cases $K_{0}=G F(3)$ or $G F(5)$ are excluded for dimension three. The only case considered by Dieudonne for index zero is the orthogonal with dimension of the vector space equal to three $[2$, p. 52$]$.

6. Remarks. The proofs outlined above give a relatively uniform treatment of all of the groups considered for infinite or sufficiently high dimensions. It is worth noting the contrast with the proofs given by Dieudonné for the finite-dimensional cases. Dieudonné also makes some use of the methods introduced by Mackey as well as methods of Schreier and van der Waerden. However he relies heavily on structure theory [3] which leads to a great multiplicity of cases. This also explains his restriction to nonzero index in the orthogonal and unitary cases since the structure theory is not available here for index zero. It also explains his restriction of the coefficient domain in the unitary case. Needless to say, there is practically no structure theory available in the infinite-dimensional cases. The proofs sketched in the preceding sections rely much more heavily on Mackey's methods and represent a fairly thorough exploitation of those methods. On the other hand, these methods break down for characteristic equal to two and extension of the isomorphism theorems to infinite dimensions for characteristic two has yet to be done. Even in finite dimensions only the full linear and symplectic groups have been considered for characteristic two [2, p. 93]. Hua, in his treatment of the 
questions considered here, uses still other methods based on direct calculation with matrices which are quite effective for low dimensions where the other methods run into difficulties.

The projective group associated with a group $G$ of linear transformations may be defined as the group $G$ modulo its center $Z$. If $G$ is one of the classical groups, then $\mathcal{Z}$ reduces to scalar multiples of the identity transformation where the scalars belong to the center of the coefficient ring. When the group $G$ is allowed to contain semi-linear transformations, it is natural to take $Z$, instead of equal to the center of $G$, equal to the group of all scalar multiples of the identity which belong to $G$. It would be desirable to extend the isomorphism theorems discussed above to the corresponding projective groups. This has been done by Dieudonné [2] for automorphisms of the linear groups in finite dimension, ${ }^{10}$ the main result being that an automorphism of the projective group is induced by an automorphism of the associated group of linear transformations (with exceptions, of course, among the low dimensions). A study of involutions in the projective group $G / Z$ leads to consideration of "projective involutions" in $G$; that is, transformations $T$ in $G$ such that $T^{2}=\zeta I \in Z$. The problem then is to characterize within the projective group $G / Z$ those elements which are determined by extremal involutions in $\mathcal{G}$. In other words, one has to distinguish, modulo $Z$, the extremal involutions from the other projective involutions in $G$. Once such a characterization of extremal involutions is obtained, the way is clear to apply the methods of the preceding sections to the projective groups. We are, as yet, unable to deal adequately with the projective groups in infinite dimensions but hope to return to this problem at another time.

It seems probable that the isomorphism theorems can be obtained for certain coefficient domains more general than division rings. An interesting case would be that of an integral domain. As another example, consider an $R$-module $\mathbb{Z}$ (that is, $\mathbb{Z}$ is an additive group which admits elements of the ring $R$ as operators) and assume given an involution in $R$. In an obvious way, one can extend the notion of a self-dual vector space to this situation and so obtain generalized symplectic and unitary groups $\mathcal{L}^{*}(\Re, R)$. The generalized unitary groups obtained in this way contain as special cases groups of the form $\mathcal{L}\left(\mathfrak{X}, \mathcal{D}, \mathfrak{X}^{*}\right)$. This fact suggests the possibility of an even more uniform treatment of the problems considered here.

${ }^{10}$ Schrier and van der Waerden also consider the projective group. 


\section{BIBLIOGRAPHY}

1. R. Baer, A theory of crossed characters, Trans. Amer. Math. Soc. vol. 54 (1943) pp. 103-170.

2. J. Dieudonné, On the automorphisms of the classical groups, Memoirs of the American Mathematical Society, no. 2, 1950.

3. - - Sur les groupes classiques, Actualités Scientifiques et Industrielles, no. $1040,1948$.

4. - Sur le socle d'un anneau et les anneaux simples infinis, Bull. Soc. Math. France vol. 71 (1943) pp. 1-30.

5. L. K. Hua, On the automorphisms of the symplectic group over any field, Ann. of Math. vol. 49 (1948) pp. 739-759.

6. - - Supplement to the paper of Dieudonne on the automorphisms of classical groups, Memoirs of the American Mathematical Society, no. 2, 1950.

7. N. Jacobson, Normal semi-linear transformations, Amer. J. Math. vol. 61 (1939) pp. $45-58$.

8. - On the theory of primitive rings, Ann. of Math. vol. 48 (1947) pp. 8-21.

9. G. Mackey, Isomorphisms of normed linear spaces, Ann. of Math. vol. 43 (1942) pp. 244-260.

10. C. E. Rickart, Isomorphic groups of linear transformations, Amer. J. Math. vol. 72 (1950) pp. 451-464.

11. - Isomorphic groups of linear transformations, II, Amer. J. Math. vol. 73 (1951) pp. 697-716.

12. O. Schreier and B. L. van der Waerden, Die Automorphismen der projektiven Gruppen, Abh. Math. Sem. Hamburgischen Univ. vol. 6 (1928) pp. 303-322.

YALE UNIVERSITY 